\title{
Genetics of obesity
}

\author{
Alfredo Martínez-Hernández ${ }^{1, *}$, Luís Enríquez ${ }^{2}$, María Jesús Moreno-Moreno ${ }^{1}$ and \\ Amelia Martí ${ }^{1}$ \\ ${ }^{1}$ Department of Physiology and Nutrition, University of Navarra, 31008 Pamplona, Spain: ${ }^{2}$ Endocrinology, \\ SEEDO, Spain
}

Submitted 27 May 2006: Accepted April 2007

\begin{abstract}
Objective: The aim was to review and update advances in genetics of obesity. Design: Analysis and interpretation of recent investigations about regulating the energy balance as well as about gene-nutrient interactions and current nutrigenomic research methods.

Background and main statements: Obesity results from a long-term positive energy balance. However, its rising prevalence in developed and developing societies must reflect lifestyle changes, since genetic susceptibility remains stable over many generations. Like most complex diseases, obesity derives from a failure of adequate homoeostasis within the physiological system controlling body weight. The identification of genes that are involved in syndromic, monogenic and polygenic obesity has seriously improved our knowledge of body weight regulation. This disorder may arise from a deregulation at the genetic level (e.g. gene transcription or altered protein function) or environmental exposure (e.g. diet, physical activity, etc.).

Conclusions: In practice, obesity involves the interaction between genetic and environmental factors.
\end{abstract}

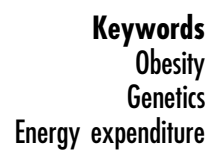

While it is clear that environmental factors play a significant role in the development of obesity, research work over the last decades has clearly documented a genetic contribution to obesity-related phenotypes as well. Obesity and its related traits represent the archetype of a common complex phenotype. The configuration of multiple genes can range from polygenic (i.e. many genes with a relatively small contribution) to oligogenic (i.e. few genes with large measurable effects often expressed on a residual polygenic background $)^{1}$. Indeed, it is this oligogenic architecture that justified all current efforts to map genes for complex phenotypes.

Prior to the mid-1990s, most of the work on genetics of human obesity had been limited to demonstrating that variation in a wide range of obesity-related phenotypes could be attributable to varying degrees to the effects of genes $^{2}$. Quantitative genetics analyses (twins, siblings and family studies) have shown significant heritability, individual's chances of being obese are increased when having obese relatives, with estimates of heritability generally ranging from $30 \%$ to $70 \%$. Different sets of monozygotic twins overfed showed differences in the degree to which excess calories were stores of fat, but the tendency towards increased adiposity within each set of twins were very similar, indicating that genetic factors play a major role in the individual susceptibility to gain weight in a specific environment ${ }^{3-5}$.
From the late 1980s through the early 1990s, segregation analysis was utilised in an effort to dissect further the genetic component underlying human obesity by attempting to detect the effects of anonymous major genes on obesity-related phenotypes. A number of these studies have reported consistent evidence of an anonymous major gene effect accounting for as much as $40 \%$ of the variation in body mass index (BMI) or fat mass in a variety of populations ${ }^{2}$.

By the early to mid-1990s, researchers began to focus on the goal of identifying the specific genes involved. Initially, such efforts to identify specific genes influencing complex traits such as those associated to obesity relied on the use of a priori selected candidates genes. Such candidates genes are selected on the basis of their perceived role or function in biochemical pathways relevant to the specific phenotype of interest (e.g. the structural gene for a circulating protein). The conservation of hypothalamic pathways in rodents and humans has certainly aided in choosing suitable candidates genes. All the spontaneously occurring obesity mutations in mice either have been found to harbour functionally relevant mutations in humans too or have led to the identification of a system/pathway in which other genes were found to be mutated. The tools recently developed allow easy determination of most gene polymorphisms from a blood sample, especially single-nucleotide polymorphism 
(SNP). This advancement opens a new era where research work devoted to study the interaction among diets, metabolic variables, disease risk factors and gene polymorphism can be carried out. According to the last available version of the Gene Obesity Map (2004) there are more than 600 genes, markers and chromosomal regions involved in body weight regulation and obesity development ${ }^{6}$.

\section{Genetics of body weight regulation}

Body weight regulation and stability depends upon an axis with three interrelated components: food intake, energy expenditure and adipogenesis, although there are still many unknown features concerning fuel homoeostasis and energy balance. There are 358 studies on obese humans reporting positive associations with 113 candidate genes. Among them, 18 genes are supported by at least five positive studies ${ }^{6}$ (Fig. 1). Here we will summarise a number of mutations in genes encoding factors regulating food/energy intake and factors implicated in energy expenditure and adiposity.

\section{Genes encoding factors regulating food/energy intake}

It was generally accepted that hypothalamic and brain stem centres are involved in the regulation of food intake and energy balance but information on the relevant regulatory factors and their genes was scarce before the last decade $^{1}$. Insulin remained the only candidate for the key role in body weight regulation for a long time. The discovery of leptin is one of the most exciting findings of the last decade. This cytokine-like peptide mainly expressed by adipocytes is now believed to be a key regulator of fat metabolism and energy intake. Leptin is the product of human homologue of mouse 'obese' gene, whose homozygous mutation caused hereditary obesity in mice (monogenic).

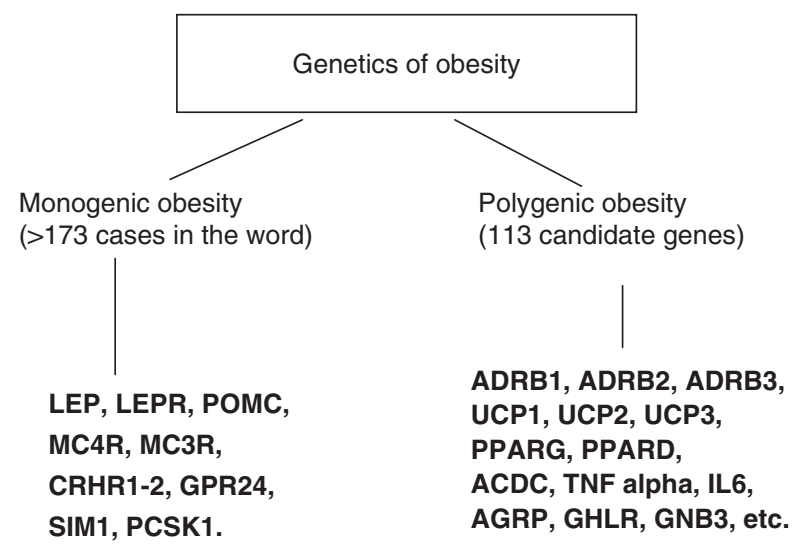

Fig. 1 Genetics of obesity
The mechanisms participating in the effects of leptin and other peptides on food intake and body weight regulation are now becoming clearer. Certain areas of the hypothalamus are rich in specific receptors binding regulatory peptides and triggering central regulatory mechanisms. Factors acting at the central nervous system level include neuropeptide Y (NPY), corticotropin-releasing hormone, proopiomelanocortin, $\alpha$-melanocyte-stimulating hormone, agouti-related protein, melanin-concentrating hormone, cocaine- and amphetamine-regulated transcript and other molecules. Interaction between them involving complex neuronal mechanisms eventually influence the behaviour and provide important links with neuroendocrine regulation of other vital functions of the organism ${ }^{2}$.

Studies in humans have failed to find leptin or any other mutant gene to be the unique 'obesity gene'. Conversely, multifactorial patterns involving actions of numerous polymorphic gene products now look more likely. Evidence is accumulating that most of these genes encoding central peptide factors as well as their receptors (leptin receptors, melanocortin receptors, NPY receptors) are polymorphic. Dominant inheritance of obesity conferred by missense, nonsense and frameshift mutations in the melanocortin 4 receptor $(M C 4 R)$ gene has been extensively reported in many populations including French, English, German, American, Italian and Spanish individuals ${ }^{2,7-10}$. It has been estimated that $1-6 \%$ of extremely obese individuals harbour functionally relevant $M C 4 R$ mutations. More than 70 mutations of the $M C 4 R$, 57 non-synonymous, 5 nonsense and 10 frameshift mutations, have been reported, many of them associated with dominant inheritance of obesity ${ }^{7,9}$. Functional studies showed that many of the missense mutations also lead to a loss-of-function of the $M C 4 R$. Meanwhile, other mutations (i.e. Thr-11-Ser, Arg-18-Cys) and two polymorphisms (Val-103-Ile, Ile-251-Leu) did not modify the function of the $M C 4 R$ in vitro ${ }^{2}$.

NPY is released from the arcuate hypothalamic nucleus in fasting or in hypoglycaemia situations, its secretion being inhibited after food intake. The Leu7Pro polymorphism in the NPY gene appears to be implicated in lipid metabolism regulation. Some works reported that carriers of the Pro 7 allele had higher NPY levels and also body fatness ${ }^{11}$.

A number of peptides synthesised along the gastrointestinal tract also affect food intake. They include ghrelin (orexigenic peptide mainly produced in the stomach), cholecystokinin (produced in the small intestine acting as a short-term satiety signal) and peptide YY3-36 (produced in the colon and suppressing appetite for up to 12 hours). Exploration of these signalling pathways has started and it is becoming clear that polymorphism in relevant genes may have important functional consequences. For the ghrelin receptor gene, two SNPs were reported: Ala204Glu and Phe279Leu, which selectively impair the constitutive activity of the receptor 
in humans leading to short stature and obesity that apparently develop during puberty ${ }^{12}$.

Moreover, the identification of relevant genes related to food preferences has just started. A novel family of 40-80 human and rodent $G$ protein-coupled receptors expressed in taste receptor cells of tongue and palate epithelia has been identified. Taste 2 receptors (T2Rs) have been shown to function as bitter taste receptor and T1Rs as putative receptor for sweet taste. There is no information on polymorphism in the T1R family genes while some SNPs in T2R have been reported ${ }^{13,14}$. Rapid progress has been made in this field to elucidate the genetic mechanism controlling formation of food preferences.

\section{Genes encoding factors implicated in energy expenditure}

The adaptive thermogenesis in humans is closely related to the active mobilisation of lipids from fat tissues and demand special interest in relation to obesity. Central neural pathways responsible for the food intake and energy expenditure regulation are tightly interconnected. The peripheral transmission of central commands to the fat stores is mediated by the sympathetic nervous system. $\beta$-adrenoceptor gene families (ADRB2, ADRB3, ADRB1) are intensively studied candidate genes in the obesity field for their participation in energy expenditure regulation.

The $\beta 2$-adrenergic receptor gene (ADRB2) encodes a major lipolytic receptor protein in human fat cells. Two common polymorphisms of the ADRB2 gene, characterised by an amino acid replacement of arginine by glycine in codon 16 ( $\operatorname{Arg} 16 G l y)$ and glutamine by glutamic acid in codon 27 ( Gln27Glu), have been explored in several diseases such as hypertension and obesity $^{2,15-18}$. A relationship between the Arg16Gly polymorphism and an altered function of the ADBR2 has been reported leading to a decreased agonist sensitivity. Meanwhile, the Gln27Glu variant was also found to be linked to obesity in some populations. In men, the $27 \mathrm{Glu}$ allele has been associated with increased BMI and subcutaneous fat and with elevated leptin and triglycerides levels, while in women, the $27 \mathrm{Glu}$ variant was reported to be linked to increased BMI, body fat mass and waistto-hip ratio ${ }^{15-18}$. However, other studies in Caucasians (Danish men, Austrian women and German subjects) found no association between the Gln27Glu variant of the $A D R B 2$ gene and obesity ${ }^{2,6}$.

The $\beta 3$-adrenergic receptor $(A D R B 3)$ protein plays a role in adipocyte metabolism mediating the rate of lipolysis in response to catecholamines and their agonists have potential anti-diabetes and anti-obesity properties ${ }^{2,19-22}$. A common polymorphism in this gene, characterised by an amino acid replacement of tryptophan by arginine at position 64 (Trp64Arg), has been identified and may be linked to lower lipolytic activity and account for lipid accumulation in the adipose tissue. A number of articles have reported the relationship between the Trp64Arg variant of the $A D R B 3$ and obesity-related phenotypes. With regard to BMI, more than nine studies have shown a statistically significant association between BMI and the Trp64Arg polymorphism in a variety of populations from 134 to 856 subjects. Besides, two metaanalyses examining the effect of this mutation on BMI have been published for Caucasian populations. One includes 2447 subjects and the significant BMI difference among carriers and non-carriers of the mutation is $0.30^{22}$. The second includes 7399 subjects but the results are negative for the association ${ }^{22}$. This polymorphism has been associated with abdominal/visceral fat obesity in several populations such as Caucasians and Japanese subjects. Similarly, several studies carried out among Mexican American, Japanese and Caucasian women have shown that carriers of the Arg allele had a higher BMI and lower reduction in visceral fat after weight $\operatorname{loss}^{2,23}$. Some authors, however, failed to reproduce the finding on $\beta$ adrenoceptors gene variants and further confirmation is required.

More recently, variants of the $A D R B 1$ gene have been studied in relation to obesity. The $A D R B 1$ is also considered a potential candidate gene for obesity because of its role in catecholamine-induced energy homoeostasis. Stimulation of $A D R B 1$, a member of G-protein-coupled receptors, mediates energy expenditure and lipolysis in adipose tissue. In the C-terminal intracellular G-protein-coupling domain of ADRB1, a polymorphism Arg389Gly has been identified, with the Arg389 allele vs. the Gly389 allele displaying enhanced adenylate cyclase activity in vitro ${ }^{24,25}$.

Whereas $\beta$-adrenoceptors participate in the regulation of adaptive thermogenesis as a component of sympathetic responses, uncoupling proteins (UCPs) are involved in the modulation of heat-generating uncoupled respiration at the mitochondrial level. They represent a family of carrier proteins localised in the inner layer of mitochondrial membranes ${ }^{22}$. There are different members: UCP1, mostly expressed in brown adipose tissue, has a role in thermogenesis, UCP2 is ubiquitously present in any tissue and UCP3 is mainly expressed in the skeletal muscle and brown adipose tissue. Their putative role as 'uncoupling proteins' has been intensively explored; like UCP1, UCP2 mediates mitochondrial proton leak releasing energy stores as heat and thereby affecting energy metabolism efficiency ${ }^{22}$. The actual functions for UCP2 and UCP3 proteins are still under investigation. It has been proposed that uncoupling proteins act as regulators of energy metabolism, they being fatty acid transmembrane transporters in the mitochondria facilitating proton exchange $^{22}$.

Moreover, a number of human studies indicated the relationship between UCP polymorphisms and exercise efficiency, resting energy expenditure, substrate oxidation, 
energy metabolism, BMI, obesity risk, type 2 diabetes risk, leptin, fat accumulation, body weight changes, physical activity and so on ${ }^{2}$. These observations have led to the consideration of UCP2 and UCP3 as candidate genes for obesity, given their function in the regulation of fuel metabolism.

Several UCP2 gene variants have been described: a G/A mutation in the promoter region $-866 \mathrm{G} / \mathrm{A}$, a valine for alanine substitution at amino acid 55 in exon 4 ( Ala $55 \mathrm{Val}$ ) and a 45 base pair insertion/deletion in the untranslated region of exon $8^{22,26,27}$. The association between these polymorphisms of the UCP2 and various aspects of obesity have been intensively studied. From the literature, it seems that allele $\mathrm{G}$ in the promoter region of $U C P 2$ increases obesity risk while it affords relative protection for type 2 diabetes ${ }^{22}$. Meanwhile the Ala55Val polymorphism has shown to be associated with increased exercise efficiency ${ }^{26}$. However, results concerning the exon 8 insertion allele of the $U C P 2$ gene have been inconsistent. While no association with obesity was observed in a number of studies conducted in several populations, significant associations between the exon 8 insertion of the UCP2 gene and BMI or fat mass or presence of obesity were found with $P$ values of $0.01,0.001$, 0.002 and $0.005^{22,27}$. A sixth study reported an association of the exon 8 insertion allele with sleeping metabolic rate $(P=0.007)^{26,27}$.

There are also several UCP3 gene variants. In linkage studies, some of them have been associated to a higher obesity risk ${ }^{22}$. Specifically, the $-55 \mathrm{C} / \mathrm{T}$ polymorphism in the promoter region of this gene has been associated with an elevated BMI, an increased level of adiposity or a greater waist-to-hip ratio ${ }^{28}$. However, other authors have not found any relationship between this polymorphism and a higher risk of obesity or changes in the metabolic rate ${ }^{28}$. Even some studies have reported an inverse correlation with BMI and the presence of $-55 \mathrm{C} / \mathrm{T}$ polymorphism ${ }^{28}$.

\section{Genes encoding factors implicated in adipogenesis}

The last group of genes acting in connection with the peripheral regulation of energy expenditure comprises the transcription factors leading to adipogenesis and adipocytes differentiation. The key actor is peroxisome proliferator-activated receptor $\gamma$, particularly the adiposespecific isoform PPARG2. In a meta-analysis examining the Pro12Ala polymorphism in 19136 subjects, a positive association with BMI was found ${ }^{22}$. In our study, the frequency of the Ala allele, similar to other Caucasian populations, was higher in obese subjects (allelic frequency 0.13 ) than in controls (0.08), suggesting that this polymorphism was associated with obesity ${ }^{29}$. There is also information on the functional role of PPARG gene variants. Some mutant proteins appear to have a reduced activity $^{22}$.

\section{Monogenic obesity}

The clinical features of human subjects with leptin (or leptin receptor), proopiomelanocortin (POMC), MC4R and proprotein convertase 1 (PC1) deficiency ${ }^{30}$ are often associated with an obese phenotype.

Congenital human leptin deficiency has been identified in subjects showing severe early-onset obesity ( 8 years and $86 \mathrm{~kg}$, or 2 years and $29 \mathrm{~kg}$ ) with intense hyperphagia and undetectable levels of serum leptin due to a frameshift mutation in the ob gene (deletion G133) in a homozygosis, which resulted in a truncated protein not secreted $^{3}$. Children with leptin deficiency had also profound abnormalities in T-cell number and function consistent with high rates of infection and childhood mortality from infections. Leptin therapy in these subjects has a major effect on appetite with normalisation of hyperphagia and reductions body weight. Leptin receptordeficient subjects were also found, with the phenotype being similar to those with leptin deficiency. The birth weight was normal, but a rapid weight gain was seen in the first months of life, with severe hyperphagia and aggressive behaviour when food was denied ${ }^{3,30}$. Basal temperature and resting metabolic rate were normal and they were normoglycemic with mildly elevated plasma insulin as reported for leptin-deficient subjects. But specifically, they also debut with mild growth retardation and impaired basal and stimulated growth hormone secretion ${ }^{3}$.

Homozygous and heterozygous subjects for mutations in POMC have been found. In neonatal life these subjects showed adrenocorticotropic hormone (ACTH) deficiency (the POMC gene encoded ACTH and other peptides), the children have red hair and pale skin due to the lack of melanocyte-stimulating hormone (MSH) action at the melanocortin-1 receptors in skin and hair follicles ${ }^{3}$. The POMC deficiency is associated with hyperphagia and early-onset obesity due to the lack of activation of the melanocortin-4 receptor.

Since 1998 many groups have reported at least 70 mutations in $M C 4 R$ mostly associated with severe early-

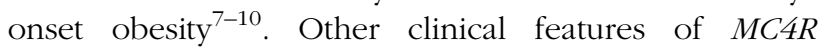
mutation carriers are hyperphagia, accelerated linear growth in children and marked increase in bone mineral density. Probands with homozygous $M C 4 R$ mutations show more severe obesity than their heterozygous relatives; thus, the mode of inheritance is codominant ${ }^{3}$. The prevalence of pathogenic $M C 4 R$ mutations varied, being the highest in obese adults selected for severe childhood obesity, suggesting that both factors, severe obesity and early age of onset, may be markers of $M C 4 R$ mutations. It has been also shown that pathogenic $M C 4 R$ mutations are more prevalent in northern European populations than in the Mediterranean or even Asian populations. The figures for prevalence are always quite low, for example 68 obese patients with pathogenic $M C 4 R$ mutations out of 
2000 or even one mutation in a sample of 3000 obese patients in another published study ${ }^{7}$. Functional analyses of these mutations allow us to classify them on the basis of their effects on receptor signalling. For instance, some authors demonstrated that mutations that caused intracellular retention of the receptor in vitro were associated with earlier age of onset and greater severity of obesity than other mutations.

The extremely low prevalence of pathogenic $M C 4 R$ mutations in the general population underlies the fact that the obesity epidemic is not an epidemic of new mutations.

Subject carriers of PC1 mutations mainly have severe early-onset obesity, impaired prohormone processing and hypocortisolaemia ${ }^{3}$. Another clinical feature is small intestine dysfunction, which may result from an erroneous maturation of propeptides within the PC1-secreting cells along the gut ${ }^{3}$.

\section{Syndromic forms of obesity}

There are about 30 rare syndromes caused by discrete genetics defects or chromosomal abnormalities with obesity as a clinical feature in association with mental retardation, dysmorphic features and organ-specific abnormalities (i.e. pleiotropic syndromes) ${ }^{3,4}$. At least four syndromes show severe hyperphagia and/or other signals of hypothalamic dysfunction, indicating a plausible origin at the level of the central nervous system. The most frequent of these syndromes is Prader-Willi syndrome (prevalence 1/25000) characterised by obesity, hyperphagia, hypotonia, mental retardation, short stature and hypogonadiotropic hypogonism. It is usually caused by lack of the paternal segment 15q11.2-q12, either through deletion of the paternal critical segment (75\%) or through loss of the entire paternal chromosome 15 with the presence of two maternal homologues in $22 \%$ of patients (uniparental maternal disomy). One suggested mediator of the obesity phenotype is ghrelin, the stomach-secreted peptide that increased appetite by interacting with $P O M C /$ $C A R T$ (cocaine- and amphetamine-regulated transcript) and NPY hypothalamic neurons whose levels are high in Prader-Willi syndrome patients ${ }^{3,4}$.

The loss of the single-minded homologue 1 (SIM1) gene in chromosome 6 has been associated with hyperphagia in syndromic obesity. This gene encoded a protein which is a regulator of neurogenesis. In humans, deletion or disruption of the SIM1 region results in either 'PraderWilli-like' phenotype or an early-onset obesity linked to hyperphagia ${ }^{3,4}$.

Albright's hereditary osteodystrophy is an autosomal dominant disorder due to mutations in GNASI, which encodes for $\alpha$-subunit of the stimulatory G protein (Gs $\alpha$ ). Maternal transmission of GNASI mutations leads to Albright's hereditary osteodystrophy (obesity, short stature, round facies, ectopic tissue ossification) plus resistance to several hormones - such as parathyroid hormone - which activate Gs in their target tissues, while paternal transmission leads only to Albright's hereditary osteodystrophy phenotype (pseudopseudohypoparathyroidism $)^{3,4}$.

The origin of obesity is more complex in Bardet-Biedl syndrome (prevalence of $\mathrm{BBS}<1 / 100000$ ). It is an autosomal recessive syndrome characterised by central obesity (75\%), polydactily, learning disabilities, rod-cone dystrophy, hypogonadism and renal abnormalities. The Bardet-Biedl syndrome is a genetically heterogenous disorder that is known to map to at least eight loci, seven of which have now been identified at the molecular level (mutations in BBS1-BBS11 genes) $^{3,4,31}$.

The molecular causes that underlie the aetiology of syndromic obesity are far more complex than that for monogenic obesity. Positional genetic strategies have led to the recent identification of several causative mutations responsible for these syndromes; however, in many cases the defective gene product is a ubiquitously expressed protein of unknown function.

\section{Genetic and dietary influences at the present time}

The interaction of functional gene polymorphisms with environmental factors (gene-environment interactions) plays a substantial role in obesity risk. Gene-environment interaction implies that in combination the effect of genotype and environment deviates from the additive or multiplicative effects of the two factors. A simple statistical approach to the analysis of gene-environment interaction when dealing with a quantitative trait (e.g. BMI) is to use the statistical test analysis of covariance, with the quantitative trait being entered as the dependent variable, genotype and environmental factors being entered as main effects and with an interaction term between the genotypic and environmental factors. If this term is statistically significant, the implication is that there is a greater or lesser additive effect and an interaction is suggested. However, this outcome could be the result of chance alone, which highlights the importance of confirmatory findings. If the $P$ value for the interaction term is not significant, the implication is that there is a lack of interaction, but it could also simply reflect the lack of power in the study to detect an effect ${ }^{2,5}$.

From a mechanistic viewpoint, interaction suggests that at the molecular level the effect or byproducts of the environmental insult modifies the molecular function of the product of the gene under observation ${ }^{33-37}$. The higher obesity risk will come from the co-existence of both genetic and environmental influences at a high scale for a given population. On one hand individuals inherited a number of gene variants in key loci and on the other they adopt a different position on the environmental spectrum of risk by the lifestyle choices they make (e.g. low-fat vs. high-fat diets, high vs. low levels of physical 
activity, etc.) Thus, while the environmental factors are modifiable, the genetic factors are not ${ }^{32}$. Understanding the gene-environment relationship is one of the big challenges that is being faced.

\section{Metbodological aspects on the study of genetics of obesity}

A complementary strategy to the candidate gene approach for the identification of obesity genes is the use of genome-wide linkage scans. This strategy involves the genotyping of families using polymorphic markers positioned across the whole genome followed by calculation of the degree of linkage of the marker to a disease trait. This approach does not rely on any pre-existing knowledge of the genes related to the disease. For the study of obesity, genome-wide linkage scans have been applied: in families, representative of general population and also in families chosen because they have an around obese proband. This latter approach is very useful for linkage analysis. In fact, the first genome scans for obesity located a quantitative trait locus (QTL) for leptin levels and fat mass at 2p21 in Mexican-Americans for the San Antonio Family Heart Study. Subsequently, more than 204 quantitative trait loci for obesity-related phenotypes were found on all but chromosome $\mathrm{Y}$ from more than 50 genome-wide scans reported since $1997^{4,6}$. Most of the genome-wide scans are based primarily on BMI measurements. Several of these QTLs have been confirmed in various studies, at least 38 QTLs showed evidence of linkage in two studies ${ }^{6}$.

Nowadays, the availability of OMICs technology represents a major event for studying the genetics of obesity $^{38-43}$. The different biomarkers at the transcript, protein and metabolite level will hopefully develop into indicative and predictive sets of molecules, their diagnostic power cannot be uncoupled from pre-determined genetic disposition. Human genetic differences appear at the level of SNPs, copy number polymorphisms and the specific combinations of alleles (haplotypes). On top of these levels of sequence variability, epigenetic phenomena, such as DNA methylation, histone acetylation and RNA interference, add to the complexity of individually different gene regulation. Besides, the high throughput offers the opportunity to genotype more than 10000 samples a day and enables genomewide association studies $^{38-43}$. DNA hybridisation arrays represent a new tool to study the association of several different polymorphisms with the development of obesity. Gene chips containing 500000 SNPs are already available allowing the identification of possible mutations in known and unknown genes related to obesity ${ }^{41}$. This technology constitutes the appropriate tool for approaching the study of those combinations of genes and mutations that are implicated in the development of obesity in humans, as well as for establishing how environmental factors affects the onset of obesity in individuals with a specific genetic background.

\section{Acknowledgements}

Sources of funding: Financial support from the University of Navarra (LE/97), Government of Navarra (Department of Health) and Spanish Government (Health and Education Departments) is gratefully acknowledged concerning the current review.

Conflict of interest declaration: Authors declare that they have no conflict of interest in relation with this material.

Authorship responsibilities: J.A.M., M.J.M. and A.M. contributed with funds from the aforementioned organism to obtain some of the reviewed results. Also, they participated in data collection, analysis and interpretation as well as in the manuscript preparation and discussion. L.E. was substantially involved in the inclusion of the scientific contents and bibliographical search as well as in the careful reading and discussion of the final version.

\section{References}

1 Loktionov A. Common gene polymorphisms and nutrition: emerging links with pathogenesis of multifactorial chronic diseases (review). The Journal of Nutritional Biochemistry 2003; 14: 426-51.

2 Marti A, Martinez JA. Genetics of obesity: gene $\times$ diet interaction. International Journal of Vitamin and Nutrition Research 2006; 76: 184-93.

3 Farooqi IS. Genetic and hereditary aspects of childhood obesity. Best Practice \& Research. Clinical Endocrinology \& Metabolism 2005; 19: 359-74.

4 Bell CG, Walley AJ, Froguel P. The genetics of human obesity. Nature Reviews. Genetics 2005; 6: 221-34.

5 Hunter DJ. Gene-environment interactions in human diseases. Nature Reviews. Genetics 2005; 6: 287-98.

6 Perusse L, Rankinen T, Zuberi A, Chagnon YC, Weisnagel SJ, Argyropoulos G, et al. The human obesity gene map: the 2004 update. Obesity Research 2005; 13: 381-490.

7 Kublaoui BM, Zinn AR. MC4R mutations - weight before screening! The Journal of Clinical Endocrinology and Metabolism 2006; 91: 1671-2.

8 Marti A, Corbalan MC, Forga L, Martinez JA, Hinney A, Hebebrand J. Presence of new mutation in the melanocortin-4 receptor in a Spanish population. International Journal of Obesity and Related Metabolic Disorders 2003; 27: 385-8.

9 Hinney A, Bettecken T, Tarnow P, Brumm H, Reichwald K, Lichtner $\mathrm{P}$, et al. Prevalence, spectrum, and functional characterization of melanocortin- 4 receptor gene mutations in a representative population-based sample and obese adults from Germany. The Journal of Clinical Endocrinology and Metabolism 2006; 91 : 1761-9.

10 Lubrano-Berthelier C, Dubern B, Lacorte JM, Picard F, Shapiro A, Zhang S, et al. Melanocortin 4 receptor mutations in a large cohort of severely obese adults: prevalence, functional classification, genotype-phenotype relationship, and lack of association with binge eating. The 
Journal of Clinical Endocrinology and Metabolism 2006; 91: 1811-18.

11 Van Rossum CT, Pijl H, Adan RA, Hoebee B, Seidell JC. Polymorphisms in the NPY and AGRP genes and body fatness in Dutch adults. International Journal of Obesity (London) 2006; 30: 1522-28.

12 Holst B, Schwartz TW. Ghrelin receptor mutations - too little height and too much hunger. The Journal of Clinical Investigation 2006; 116: 637-41.

13 Hinrichs AL, Wang JC, Bufe B, Kwon JM, Budde J, Allen R, et al. Functional variant in a bitter-taste receptor (hTAS2R16) influences risk of alcohol dependence. American Journal of Human Genetics 2006; 78: 103-11.

14 Kim UK, Jorgenson E, Coon H, Leppert M, Risch N, Drayna D. Positional cloning of the human quantitative trait locus underlying taste sensitivity to phenylthiocarbamide. Science 2003; 299: 1221-5.

15 Macho-Azcarate T, Marti A, Gonzalez A, Martinez JA, Ibanez J. Gln27Glu polymorphism in the beta2 adrenergic receptor gene and lipid metabolism during exercise in obese women. International Journal of Obesity and Related Metabolic Disorders 2002; 26: 1434-41.

16 Macho-Azcarate T, Calabuig J, Marti A, Martínez JA. A maximal effort trial in obese women carrying the beta2adrenoceptor Gln27Glu polymorphism. Journal of Physiology and Biochemistry 2002; 58: 103-8.

17 Corbalán MS, Marti A, Forga L, Martinez-Gonzalez MA, Martinez JA. The 27 Glu polymorphism of the beta2adrenergic receptor interacts with physical activity influencing obesity risk among female subjects. Clinical Genetics 2002; 61: 305-7.

18 Corbalan MS, Marti A, Forga L, Martinez-Gonzalez MA, Martinez JA. Beta(2)-Adrenergic receptor mutation and abdominal obesity risk: effect modification by gender and HDL-cholesterol. European Journal of Nutrition 2002; $\mathbf{4 1}$ : 114-18.

19 Marti A, Corbalan MS, Martinez-Gonzalez MA, Martinez JA. TRP64ARG polymorphism of the beta3-adrenergic receptor gene and obesity risk: effect modification by a sedentary lifestyle. Diabetes, Obesity \& Metabolism 2002; 4: 428-30.

20 Corbalan MS, Marti A, Forga L, Martinez-Gonzalez MA, Martinez JA. The risk of obesity and the Trp64Arg polymorphism of the beta (3)-Adrenergic receptor: effect modification by age. Annals of Nutrition and Metabolism 2002; 46: 152-8.

21 Ochoa MC, Marti A, Azcona C, Chueca M, Oyarzabal M, Grupo de Estudio Navarro de Obesidad Infantil (GENOI), et al. Gene-gene interaction between PPARG2 and ADR beta 3 increases obesity risk in children. International Journal of Obesity and Related Metabolic Disorders 2004; 28(Suppl. 3): S37-41.

22 Ochoa Mdel C, Marti A, Martinez JA. [Obesity studies in candidate genes]. Medicina Clinica (Barcelona) 2004; 122: 542-51.

23 Park HS, Kim Y, Lee C. Single nucleotide variants in the beta2-adrenergic and beta3-adrenergic receptor genes explained $18.3 \%$ of adolescent obesity variation. Journal of Human Genetics 2005; 50: 365-9.

24 Li S, Chen W, Srinivasan SR, Boerwinkle E, Berenson GS. Influence of lipoprotein lipase gene Ser447Stop and beta(1)-adrenergic receptor gene Arg389Gly polymorphisms and their interaction on obesity from childhood to adulthood: the Bogalusa Heart Study. International Journal of Obesity (London) 2006; 30(8): 1183-8.

25 Linne Y, Dahlman I, Hoffstedt J. beta1-Adrenoceptor gene polymorphism predicts long-term changes in body weight. International Journal of Obesity (London) 2005; 29: 458-62.
26 Marti A, Corbalan MS, Forga L, Martinez-Gonzalez MA, Martinez JA. Higher obesity risk associated with the exon- 8 insertion of the UCP2 gene in a Spanish case-control study. Nutrition 2004; 20: 498-501.

27 Zurbano R, Ochoa MC, Moreno-Aliaga MJ, Martinez JA, Marti A, Grupo de Estudio Navarro de la obesidad infantil. [Influence of the $-866 \mathrm{G} / \mathrm{A}$ polymorphism of the UCP2 gene on an obese pediatric population]. Nutricion Hospitalaria 2006; 21: 52-6.

28 Alonso A, Marti A, Corbalan MS, Martinez-Gonzalez MA, Forga L, Martinez JA. Association of UCP3 gene $-55 \mathrm{C}>\mathrm{T}$ polymorphism and obesity in a Spanish population. Annals of Nutrition and Metabolism 2005; 49: 183-8.

29 Marti A, Corbalan MS, Martinez-Gonzalez MA, Forga L, Martinez JA. CHO intake alters obesity risk associated with Pro12Ala polymorphism of PPARgamma Gene. Journal of Physiology and Biochemistry 2002; 58: 219-20.

30 Zurbano R, Ochoa MC, Moreno-Aliaga MJ, Marti A. Estudios sobre obesidad de origen monogénico en humanos. Revista Española de Obesidad 2004; 2: 269-78.

31 Chiang AP, Beck JS, Yen HJ, Tayeh MK, Scheetz TE, Swiderski RE, et al. Homozygosity mapping with SNP arrays identifies TRIM32, an E3 ubiquitin ligase, as a Bardet-Biedl syndrome gene (BBS11). Proceedings of the National Academy of Sciences USA 2006; 103: 6287-92.

32 Marti A, Moreno-Aliaga MJ, Hebebrand J, Martinez JA. Genes, lifestyles and obesity. International Journal of Obesity Related Metabolic Disorders 2004; 28(Suppl. 3): S29-36.

33 Marti A, Razquin C, Martinez JA. Papel de las interaciones genes-nutrientes en el desarrollo de la obesidad. Revista Española de Obesidad 2006; 4: 86-96.

34 Luan J, Browne PO, Harding AH, Halsall DJ, O'Rahilly S, Chatterjee VK, et al. Evidence for gene-nutrient interaction at the PPARgamma locus. Diabetes 2001; 50: 686-9.

35 Memisoglu A, Hu FB, Hankinson SE, Manson JE, De Vivo I, Willett WC, et al. Interaction between a peroxisome proliferator-activated receptor gamma gene polymorphism and dietary fat intake in relation to body mass. Human Molecular Genetics 2003; 12: 2923-9.

36 Nieters A, Becker N, Linseisen J. Polymorphisms in candidate obesity genes and their interaction with dietary intake of n-6 polyunsaturated fatty acids affect obesity risk in a sub-sample of the EPIC-Heidelberg cohort. European Journal of Nutrition 2002; 41: 210-21.

37 Moreno-Aliaga MJ, Santos JL, Marti A, Martinez JA. Does weight loss prognosis depend on genetic make-up? Obesity Reviews 2005; 6: 155-68.

38 Moreno-Aliaga MJ, Marti A, Garcia-Foncillas J, Martinez JA. DNA hybridization arrays: a powerful technology for nutritional and obesity research. British Journal of Nutrition 2001; 86: 119-22.

39 Hebebrand J, Friedel S, Schauble N, Geller F, Hinney A. Perspectives: molecular genetic research in human obesity. Obesity Reviews 2003; 4: 139-46.

40 Marti A, Moreno-Aliaga MJ, Zulet A, Martinez JA. [Advances in molecular nutrition: nutrigenomics and/or nutrigenetics]. Nutricion Hospitalaria 2005; 20: 157-64.

41 Herbert A, Gerry NP, McQueen MB, Heid IM, Pfeufer A, Illig $\mathrm{T}$, et al. A common genetic variant is associated with adult and childhood obesity. Science 2006; 312: 279-83.

42 Kussmann M, Raymond F, Affolter M. OMICS-driven biomarker discovery in nutrition and health. Journal of Biotechnology 2006; 124: 758-87.

43 Kaput J. Decoding the pyramid: a systems-biological approach to nutrigenomics. Annals of The New York Academy of Sciences 2005; 1055: 64-79. 